This item was submitted to Loughborough's Research Repository by the author.

Items in Figshare are protected by copyright, with all rights reserved, unless otherwise indicated.

\title{
Video analysis of the deformation and effective mass of gymnastics landing mats
}

PLEASE CITE THE PUBLISHED VERSION

http://dx.doi.org/10.1249/01.mss.0000175053.81453.aa

PUBLISHER

(c) American College of Sports Medicine

VERSION

AM (Accepted Manuscript)

LICENCE

CC BY-NC-ND 4.0

REPOSITORY RECORD

Pain, Matthew T.G., Chris Mills, and Maurice R. Yeadon. 2019. "Video Analysis of the Deformation and Effective Mass of Gymnastics Landing Mats". figshare. https://hdl.handle.net/2134/7084. 
This item was submitted to Loughborough's Institutional Repository (https://dspace.lboro.ac.uk/) by the author and is made available under the following Creative Commons Licence conditions.

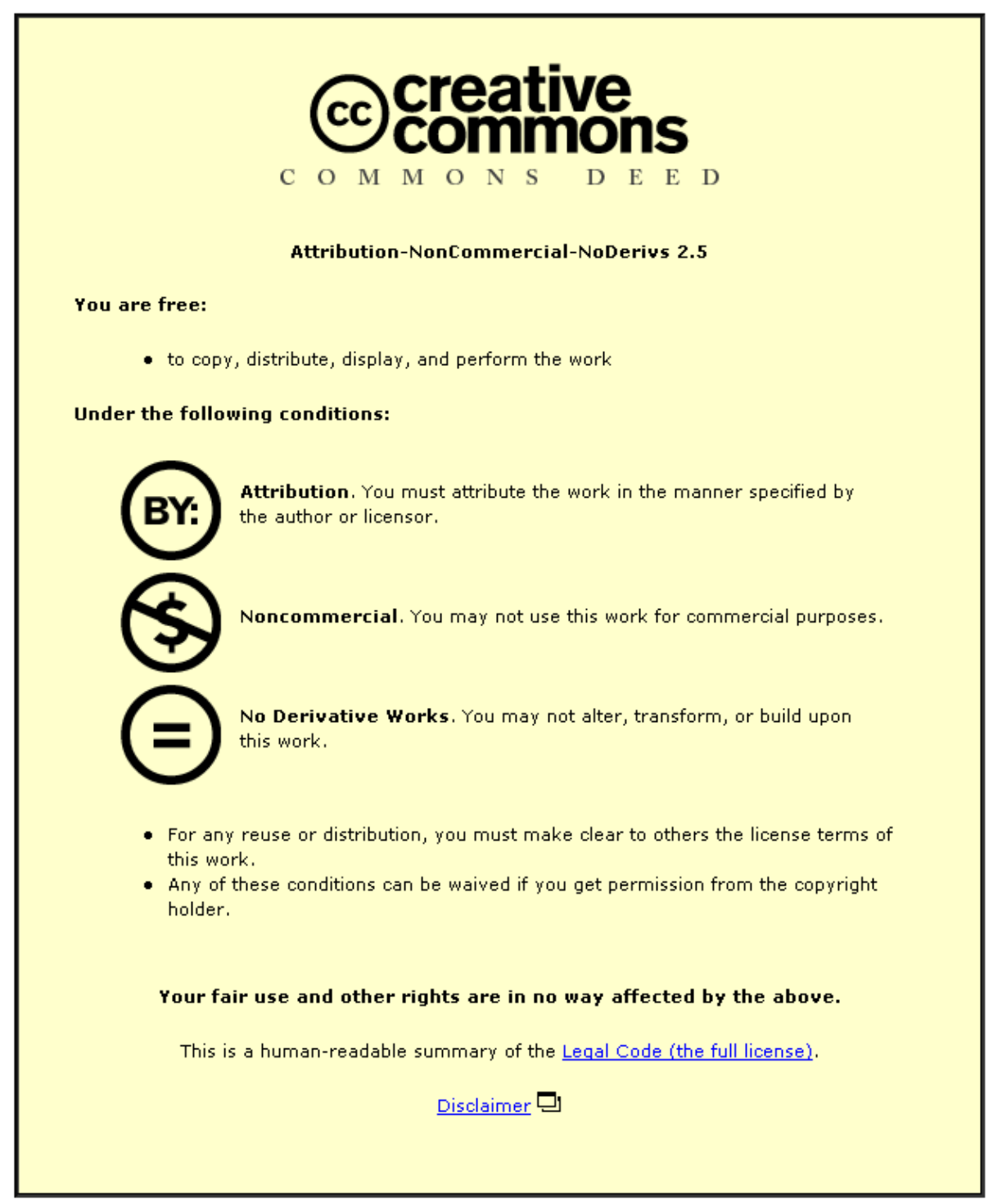

For the full text of this licence, please go to: http://creativecommons.org/licenses/by-nc-nd/2.5/ 


\title{
Video analysis of the deformation and effective mass of gymnastics landing mats
}

\author{
Matthew T.G. Pain, Chris Mills and Maurice R. Yeadon \\ School of Sport and Exercise Sciences, Loughborough University, Loughborough, United Kingdom.
}

\begin{abstract}
Introduction: Landing mats that undergo a large amount of area deformation are now essential for the safe completion of landings from dismounts and vaults in gymnastics. The aim of this paper is to determine the effective mass, shock transmission time and deformation characteristics of a mat during impacts using high-speed video and hence improve the accuracy of measuring foot / mat contact forces during landing. To this end the same variables need to be accurately assessed using accelerometer and force plate data so that the high-speed video method can be validated.

Methods: A $24 \mathrm{~kg}$ impactor with an attached accelerometer was dropped onto the sample mat from various heights. The surface deformation of the mat was recorded using high-speed video and force data were obtained from a force plate beneath the mat.

Results: Impact velocities ranged from $4.3 \mathrm{~ms}^{-1}$ to $6.5 \mathrm{~ms}^{-1}$ resulting in maximum vertical deformations between $0.088 \mathrm{~m}$ and $0.118 \mathrm{~m}$ with corresponding volume deformation estimates ranging from $0.030 \mathrm{~m}^{3}$ to $0.044 \mathrm{~m}^{3}$. The delay between accelerometer and force plate readings at initial contact was approximately $7 \mathrm{~ms}$ whereas the delay between peak acceleration and peak force was $3 \mathrm{~ms}$. The peak acceleration calculated from the video data was within $2.5 \%$ of that recorded via the accelerometer. The effective mass of the mat being accelerated corresponded to a force that ranged from $481 \mathrm{~N}$ to $930 \mathrm{~N}$ and this cannot be ignored as it accounts for up to $12 \%$ of the peak force.

Conclusions: The acceleration estimates obtained from the high-speed video were combined with the effective mass estimates from the volume calculation to give peak calculated forces at the bottom of the mat to within $-1.1 \%$ to $+3.7 \%$ of the force recorded via the force plate. The use of high-speed video can be used to give data of sufficient accuracy for measuring foot / mat contact forces in gymnastics landings.
\end{abstract}

\section{INTRODUCTION}

Artificial sports surfaces are designed to allow elastic deformation, which can enhance performance and / or reduce loading (6). When loading is too large internal structures may become damaged (2), so a compromise must be reached between performance enhancement and injury reduction. To this end a variety of surfaces have been developed which are commonly assigned to one of two groups: point-elastic surfaces that distribute forces over a small area, and area-elastic surfaces that react to a local force by deforming over a relatively large area (3). Two common examples of area-elastic surfaces are sprung wooden floors and landing mats.

Landing mats that undergo a large amount of area deformation are now essential for landings from dismounts and vaults in gymnastics. By reducing the loading on the gymnast the mats enhance performance by allowing the gymnast to perform more complex skills which in turn reduces the risk of injury. Landing mats are bulky, have a number of component layers, transmit forces relatively slowly and undergo large area-viscoelastic deformations. This introduces a number of potential problems related to the mat's construction, the dynamics of the mat whilst undergoing impact and the mat / force plate interaction. A significant amount of mat mass may be accelerated during impact and it will take a finite time for the force at the surface of the mat to be detected beneath the mat via a force plate. This time is the shock wave propagation time and is evident in other elastic materials (9). It has 
also been shown that elastic surfaces on force plates introduce $20 \mathrm{~Hz}$ or higher frequency oscillations into the force data during impacts (7). These are important factors to consider when dealing with area deformation, especially when large volumes of material are involved, as the forces of interest to the biomechanist are normally those acting at the mat / gymnast interface. These mat / gymnast interface forces differ from those at the mat / force plate interface due to the slow transmission of force through the material and the non-uniform acceleration of mat mass. If detailed analysis of the gymnast-mat interaction during landing is to be investigated, the mechanical response of the area-elastic mat in such loading regimes needs to be determined accurately.

Research on area-elastic surfaces has been performed using material tests $(4,5,8$, 11) and subject tests $(3,5,11,14)$. Material tests have usually involved the use of accelerometers attached to the mass being dropped $(4,5,8)$ or the use of a force plate beneath the landing surface $(4,11)$. The masses used have ranged from $5.5 \mathrm{~kg}(11)$ to $20 \mathrm{~kg}(4)$ and have been dropped from various heights to assess the cushioning properties of the landing mats. Subject tests have involved the participant dropping from a height onto the landing mat with a force plate beneath the mat to record the ground reaction forces $(10,11)$.

In many situations, such as in competition, it is not possible to use accelerometers or force plates. High-speed video has been used to measure vertical deformation of wooden landing surfaces but has not previously been use to assess the deformation of matted surfaces. The vertical deformation, area-elasticity and frequency response of unloaded wooden surfaces has been assessed with subject tests using high-speed cameras $(3,14)$. These studies concluded that the method was suitable for the assessment of area-elastic wooden sports surfaces with accuracies of $0.1 \mathrm{~mm}$ to $0.12 \mathrm{~mm}$. Gymnastics landing mats deform over larger areas and with greater vertical displacement than wooden floors. This may be why the use of video has been restricted to wooden floors as the increased deformation may obstruct surface markers at maximum deformation.

If high-speed video can be used to calculate the key characteristics of the impact it is possible video could be used in a competition environment where force and accelerometer instrumentation is not an option. It may also be possible to analyse different competition mats using high-speed video and compare the forces above and below the mat with those of landing mats tested in laboratory conditions. Understanding a landing mat's deformation behaviour may help the assessment of injury potential. Variables such as effective mass could be used to calculate the forces involved in deforming different mats and may therefore be used to reduce injuries associated with landing. These variables could also be combined with the foot acceleration, stiffness and damping characteristics of the mat materials or a simple mass-spring model to examine the inverse dynamics of the system and the force at the mat's surface.

The aim of this paper is to determine the effective mass, shock transmission time and deformation characteristics of a mat during impacts using high-speed video and hence improve the accuracy of measuring foot / mat contact forces during landing. To this end the same variables need to be accurately assessed using accelerometer and force plate data so that the high-speed video method can be validated.

\section{METHODS}

The testing equipment consisted of an impactor, sample mat, force plate, two highspeed video cameras and two accelerometers. A custom built impactor of mass $24 \mathrm{~kg}$ and contact area $0.25 \mathrm{~m}$ by $0.25 \mathrm{~m}$ was dropped vertically onto the centre of the sample mat from various heights $(1.03 \mathrm{~m}$ to $2.15 \mathrm{~m}$ ) producing measured impact velocities between $4.3 \mathrm{~m} / \mathrm{s}$ and 
$6.5 \mathrm{~m} / \mathrm{s}$ which were within the range of landing velocities reported in the literature for landings $(12,13)$. The impactor size was designed to match the area covered by an average gymnast's feet when hip width apart. The distance between the gymnast's feet in the landing configuration was only between 8 and $10 \mathrm{~cm}$. High speed video of gymnasts landing showed that the mat appeared to deform in an area-elastic manner with the same depression between the feet as under the feet. A single area of contact for the impactor was chosen for ease of experimental procedure since it made no difference to the deformation. The mass of the impactor was selected to give the same loading characteristics as a gymnast produced when landing on the mat from the same height (Figure 1). A male gymnast (mass $72 \mathrm{~kg}$ ) performed a competition style landing (minimal deductions as scored by F.I.G.) onto the sample-landing mat from a height of approximately $1.56 \mathrm{~m}$. (5.5 m/s vertical impact velocity). The impactor was released from the same drop height and the mass of the impactor increased with each test until the impulse, vertical force and rate of force production for the impactor matched the subject test.

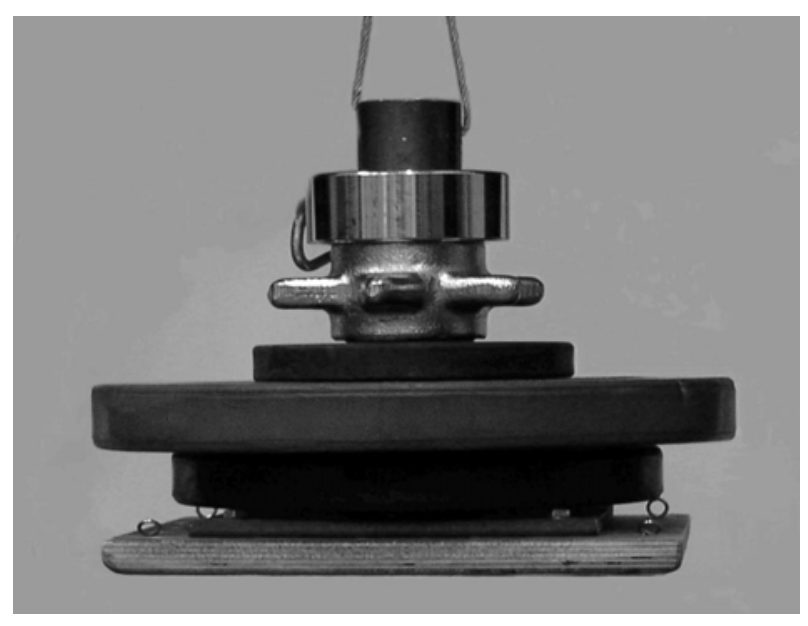

Figure 1. Custom impactor loaded with weights.

A Kistler force plate (9281B12) sampling at $1000 \mathrm{~Hz}$ was set to trigger at a level of $25 \mathrm{~N}$ with a $10 \%$ pre-trigger and a collection time of five seconds. When the force plate was triggered a signal was sent to the cameras and the accelerometers which all sampled at 1000 Hz. Two Phantom (V5) high-speed cameras (Vision Research Inc.) were used to record the impact trials. The cameras were set with a $763 \mathrm{~ms}$ post trigger so that 237 frames were recorded prior to the trigger. An accelerometer (PCB Piezotronics) was aligned vertically and was firmly attached to the impactor. All data were synchronised to within one millisecond. A trial was recorded if the impactor landed flat on the mat with minimal rotation during impact. This was determined visually and the deviation from the vertical was calculated from the digitised data $\left(\right.$ mean $=2.6^{\circ}, \mathrm{SD}=0.87^{\circ}$ ). This gave a non-vertical alignment error in the acceleration measurements of less than $0.2 \%$. Following data collection a total of five trials representing impact velocities throughout the test range were selected for further analysis.

The sample landing mat for this study was based on an official F.I.G. competition landing mat and was custom-built by the manufacturer 'Continental Sports Ltd'. The sample mat comprised two layers, an upper high density foam layer $5 \mathrm{~cm}$ thick a lower low density foam layer $15 \mathrm{~cm}$ thick. The mat had mass $6.1 \mathrm{~kg}$, measured $0.90 \mathrm{~m}$ long by $0.60 \mathrm{~m}$ wide by $0.20 \mathrm{~m}$ deep and was surrounded by a custom-built wooden frame, which was designed to constrain the landing mat so that it behaved more like a full size landing mat. Restraining the mat horizontally also prevented any slipping of the mat on the force plate. The wooden frame 
was bolted to a rigid frame that in turn was bolted to the force plate to ensure that all forces were transferred directly to the force plate during impact. To assess the area deformation of the mat 28 (5 mm diameter) markers were attached to its surface arranged in four rows of six with an additional four markers located at the corners of the mat (Figure 2).
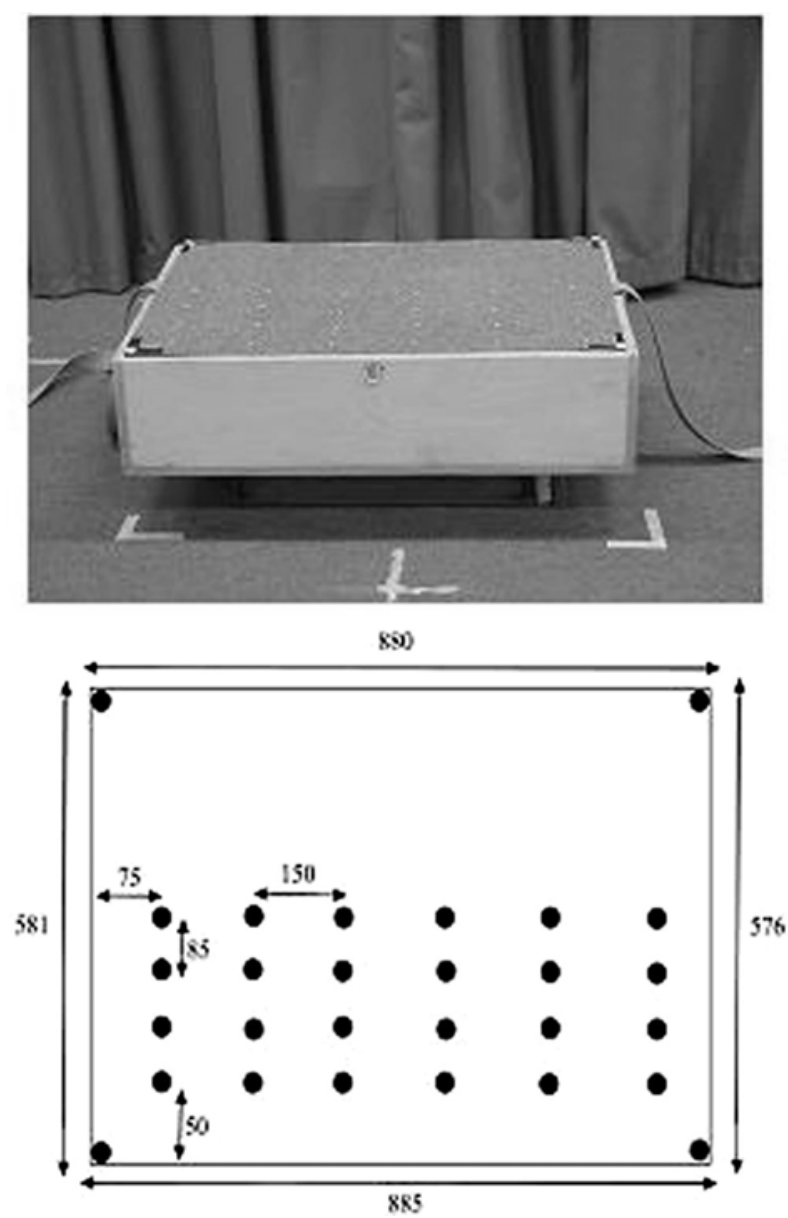

Figure 2. The sample landing mat and marker placement (distances in $\mathrm{mm}$ ).

Following data collection a total of five vertical trials were selected for further analysis. All markers on the landing mat were digitised manually to obtain displacement data. Two additional markers on the impactor were also digitised manually to determine impact velocity for each trial and maximum vertical displacement of the impactor. The KineMat DLT reconstruction MatLab program was used to reconstruct the three-dimensional coordinates of the markers throughout the impact tests (1). Prior to the impact trials a calibration structure comprising 20 markers that spanned the volume of the mat was video recorded and digitised. Ten calibration points were used to determine the 11 DLT parameters and the remaining 10 points were reconstructed to within $1 \mathrm{~mm}$ of their measured locations. The 24 markers on the mat's surface were used to calculate the volume of the mat deformed during the impact.

The shock transmission time was estimated as the difference from the time the accelerometer first detected a change in acceleration to the time when the force plate was triggered. Subsequently all data were filtered at $20 \mathrm{~Hz}$ (7) using a Butterworth $2^{\text {nd }}$ order low pass filter prior to further analysis. The acceleration data derived from the video displacement data via double differentiation was compared with that from the accelerometer. The acceleration data from the accelerometer (filtered and unfiltered) and video (filtered) were used to calculate the mat's effective mass. Using the force from the force plate and the 
acceleration from the accelerometer or video the effective mass (m) of the mat was calculated using Newton's Second Law:

$$
\mathrm{F}=\left(\mathrm{M}_{\mathrm{i}}+\mathrm{m}\right) \mathrm{a}
$$

where:

$\mathrm{M}_{\mathrm{i}}=$ mass of the impactor $(24 \mathrm{~kg})$

$\mathrm{m}=$ effective mat mass

$\mathrm{F}=$ force from force plate

$\mathrm{a}=$ acceleration from either accelerometer or video

The effect of filtering on the force and acceleration data caused a percentage drop in peak force of $14.5 \%$ and a drop in peak acceleration of $11.7 \%$. Therefore the force and acceleration data were corrected for this and the corrected effective mat mass was recalculated using:

$$
1.145 \mathrm{~F}=\left(\mathrm{M}_{\mathrm{i}}+\mathrm{m}\right) 1.117 \mathrm{a}
$$

Two methods were used to determine the mat's deformation volume. Method 1 is suitable for the small sample mat as it folded or creased along its short axis to some extent as the deformation reached the edge. Method 2 is suitable for a larger competition mat that would not have edge effects. Method 1 estimated volume at maximum mat deformation by assuming that a rectangular prism the length of the impactor and width of the mat depressed the mat to its maximum deformation. Either side of the rectangular prism two triangular prisms represented the deformation volume from the rectangular prism to the edge of the mat (Figure 3a).

where:

$$
\mathrm{V}=\mathrm{L}_{\mathrm{I}} \mathrm{WD}+0.5\left(\mathrm{~L}_{\mathrm{M}}-\mathrm{L}_{\mathrm{I}}\right) \mathrm{WD}
$$

$\mathrm{V}=$ volume estimate

$\mathrm{L}_{\mathrm{I}}=$ impactor length / width $(0.25 \mathrm{~m})$

$\mathrm{W}=$ mat width $(0.60 \mathrm{~m})$

$\mathrm{D}=$ vertical depression

$\mathrm{L}_{\mathrm{M}}=$ mat length $(0.90 \mathrm{~m})$

Method 2 assumed that a cuboid the length and width of the impactor depressed the mat to its maximum deformation. Surrounding the impactor four triangular prisms represented the deformation volume from the cuboid to the edge of the mat (Figure 3b).

where:

$$
\mathrm{V}=8 / 3 \mathrm{bcD}+2 \mathrm{~L}_{\mathrm{I}} \mathrm{bD}+\mathrm{L}_{\mathrm{I}}^{2} \mathrm{D}
$$

$\mathrm{b}=\left(\mathrm{L}_{\mathrm{M}}-\mathrm{L}_{\mathrm{I}}\right) / 2$

$\mathrm{c}=\left(\mathrm{W}-\mathrm{L}_{\mathrm{I}}\right) / 2$ 


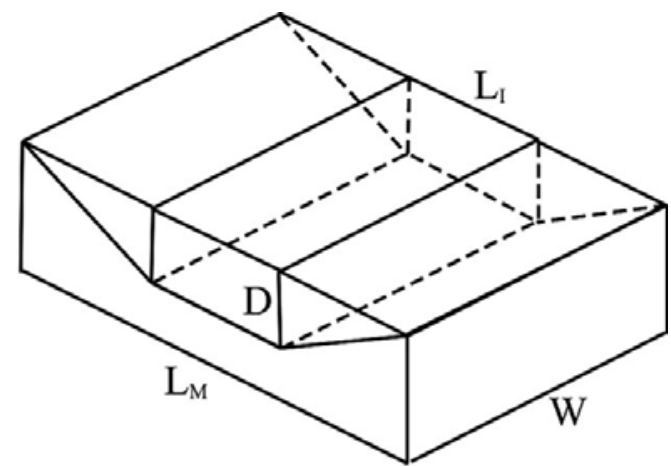

(a)

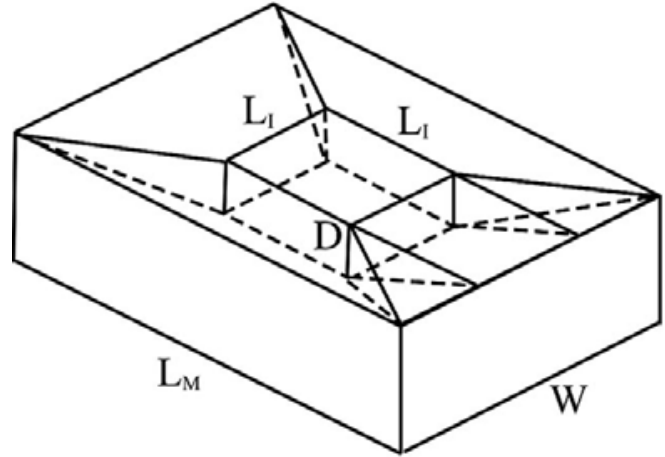

(b)

Figure 3. Estimates of mat deformation volume using two methods of calculation: (a) Method 1, (b) Method 2.

The effective mass represents the mass that would have to be accelerating at the measured acceleration to give the measured force. In the limiting case of a translating rigid body the effective mass and the real mass are equivalent, as the acceleration measured at any point equals the acceleration of the centre of mass. For a uniform body undergoing uniform compression the effective mass would equal half the real mass of the body, as the centre of mass acceleration is half the top surface acceleration. The landing mat is neither a uniform body nor does it undergo uniform compression when it deforms. Until the shock transmission includes all of the mat volume the effective mass can change. After this the effective mass should remain constant.

Video data were used to calculate the effective mass. The area of deformation of the mat, the mass of the mat layers and the shape of the deformation were used. These calculations accounted for the fact that the acceleration at the surface would not be uniform and was only measured at the point of impact. Two different assumptions of how the different density layers of the mat deformed were employed with the measurements for volume calculation Method 1. Assuming that both layers compressed equally gave equation 5, while assuming that the top layer remained uncompressed and compressed the softer layer below gave equation 7. These equations simplify leading to expressions for the effective mass (equations 6 and 8). In both cases the mat surface immediately below the impactor was accelerating at the same rate as the impactor and the average acceleration of the mat surface making up a triangular prism was assumed to be half that of the impactor. This assumption was based on one end of the prism accelerating at the same rate as the impactor and the other end remaining stationary. This gives a mean acceleration of half that of the impactor. 


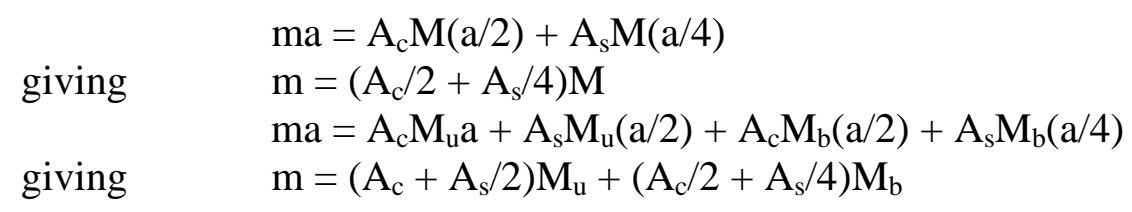

where:

$\mathrm{A}_{\mathrm{c}}=$ area of rectangular prism as a percentage of mat area

$\mathrm{A}_{\mathrm{s}}=$ area of triangular prism as a percentage of mat area

$\mathrm{M}=$ total mat mass

$\mathrm{M}_{\mathrm{u}}=$ mass of upper dense layer of mat

$\mathrm{M}_{\mathrm{b}}=$ mass of bottom soft layer of mat

$\mathrm{a}=$ acceleration measured at impactor

The force at the top of the mat was estimated using the acceleration from the video and the impactor mass. The effective mass of the mat estimated using video combined with the video acceleration produced an estimate of the force due to the acceleration of mat mass. These two forces added gave an estimate of the force beneath the landing mat and could be compared with that recorded via the force plate.

$\mathrm{M}_{\mathrm{i}}=$ mass of the impactor

$$
\begin{aligned}
& \mathrm{R}_{2}-\mathrm{M}_{\mathrm{i}} \mathrm{g}=\mathrm{M}_{\mathrm{i}} \mathrm{a} \\
& \mathrm{R}_{1}-\left(\mathrm{M}_{\mathrm{i}}+\mathrm{m}\right) \mathrm{g}=\left(\mathrm{M}_{\mathrm{i}}+\mathrm{m}\right) \mathrm{a}
\end{aligned}
$$

$\mathrm{g}=$ acceleration due to gravity

$\mathrm{a}=$ acceleration of mat's surface

$\mathrm{m}=$ effective mass of mat

$\mathrm{R}_{1}=$ force at bottom of mat

$\mathrm{R}_{2}=$ force at top of mat

\section{RESULTS}

The impact velocities ranged from $4.3 \mathrm{~ms}^{-1}$ to $6.5 \mathrm{~ms}^{-1}$ while the maximum vertical deformation ranged from $0.088 \mathrm{~m}$ to $0.118 \mathrm{~m}$ corresponding to volume deformations ranging from $0.030 \mathrm{~m}^{3}$ to $0.044 \mathrm{~m}^{3}$. The total volume of the sample landing mat was $0.108 \mathrm{~m}^{3}$. Estimating the maximum deformation volume using Method 2 resulted in about $10 \%$ more volume than using Method 1 (Table 1).

\begin{tabular}{|c|c|c|c|c|}
\hline Trial & $\begin{array}{l}\text { Impact Velocity } \\
\left(\mathrm{m} \cdot \mathrm{s}^{-1}\right)\end{array}$ & $\begin{array}{l}\text { Vertical Deformation } \\
\qquad(\mathrm{m})\end{array}$ & $\begin{array}{l}\text { Volume Estimate } \\
\left(\mathrm{m}^{3}\right)(\text { method } 1)\end{array}$ & $\begin{array}{l}\text { Volume Estimate } \\
\left.\left(\mathrm{m}^{3}\right) \text { (method } 2\right)\end{array}$ \\
\hline 1 & 4.30 & 0.088 & 0.030 & 0.033 \\
\hline 2 & 4.80 & 0.099 & 0.034 & 0.037 \\
\hline 3 & 5.25 & 0.100 & 0.034 & 0.038 \\
\hline 4 & 5.75 & 0.113 & 0.038 & 0.043 \\
\hline 5 & 6.50 & 0.118 & 0.040 & 0.044 \\
\hline
\end{tabular}

Table 1. Vertical drop test results: maximum deformation characteristics

At maximum deformation of the landing surface during a vertical impact at $5.25 \mathrm{~ms}^{-1}$ or higher the entire surface was displaced (Figure 4). 


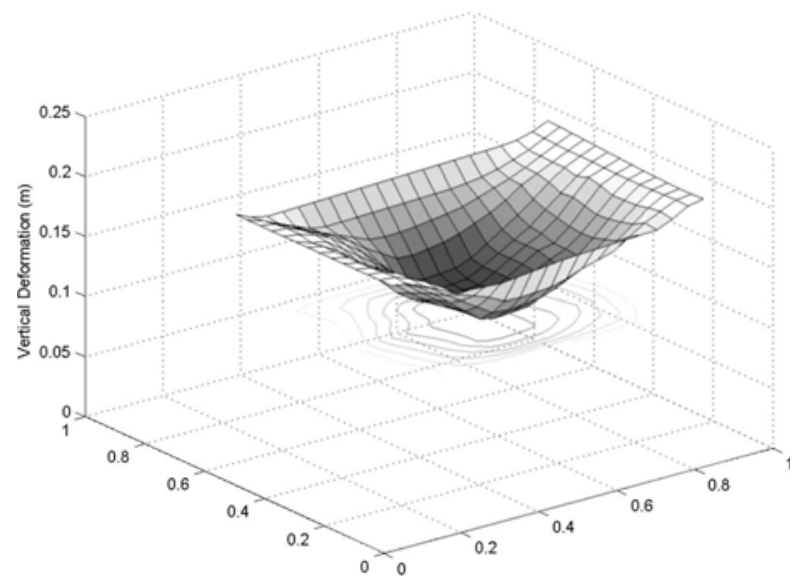

Figure 4. Surface deformation in metres at peak vertical deformation during trial 3.

A comparison of the force (via force plate) and acceleration (via accelerometer) data in Figure 5 shows the shock transmission time in trial 3; the force and acceleration magnitudes have been scaled. The delay in detecting initial contact with the accelerometer and the force plate across all trials was $7.4 \pm 0.5 \mathrm{~ms}$ whereas the delay between the peak acceleration and the peak force was $3.1 \pm 0.4 \mathrm{~ms}$.

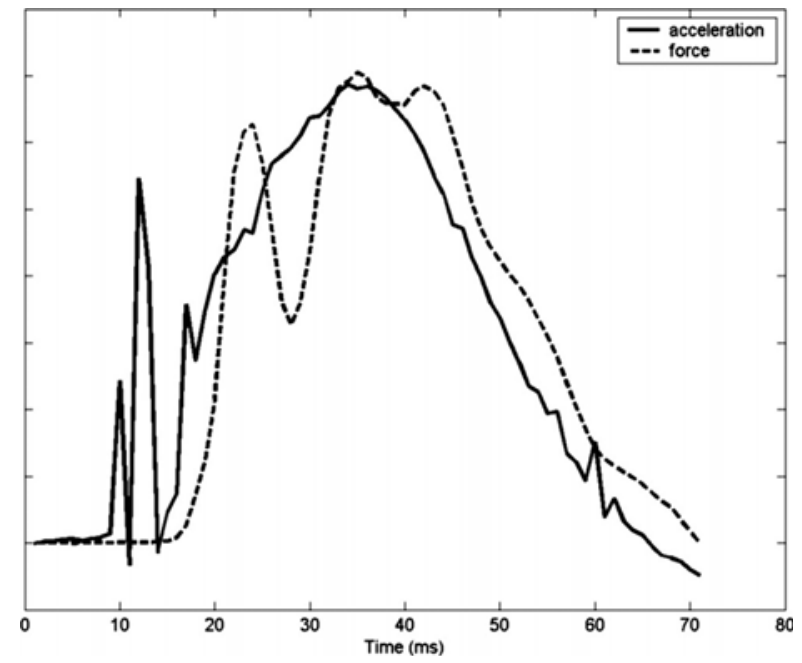

Figure 5. A comparison of force measurements taken at the mat-ground interface with accelerometer measurements of the impactor.

The time difference between the force plate trigger signal and the detection of initial impact via the accelerometers and visual detection via high-speed video ranged from $4-8 \mathrm{~ms}$ (Table 2). The time differences were not due to the force plate being triggered at $25 \mathrm{~N}$ since the trigger level caused less than 1 ms delay. 
Table 2. Impact detection times from accelerometer and video in ms

Impact detection time (ms)

Trial

Acceleromater

Video

\begin{tabular}{|c|c|c|}
\hline 1 & -7 & -4 \\
\hline 2 & -8 & -4 \\
\hline 3 & -7 & -5 \\
\hline 4 & -7 & -5 \\
\hline 5 & -8 & -6 \\
\hline
\end{tabular}

Force plate trigger, time zero

The peak filtered accelerations from the accelerometer and video were similar. The difference between the two accelerations ranged from 2 to $11 \mathrm{~ms}^{-2}$ (Table 3).

Table 3. Filtered peak acceleration data

Trial Acceleromater $\left(\mathrm{m} . \mathrm{s}^{-2}\right) \quad$ Video $\left(\mathrm{m} . \mathrm{s}^{-2}\right)$

\begin{tabular}{|l|l|l|}
\hline 1 & 192 & 185 \\
\hline 2 & 226 & 224 \\
\hline 3 & 241 & 238 \\
\hline 4 & 280 & 274 \\
\hline 5 & 289 & 300 \\
\hline
\end{tabular}

The effect of filtering on the effective mass calculation is shown in Table 4. The average percentage drop in force from unfiltered to filtered data was $14.5 \%$. The average percentage drop in acceleration from unfiltered to filtered data was $11.7 \%$.

Table 4. Effective mass of mat at peak deformation calculated using filtered force data in conjunction with either filtered accelerometer or video data, the corrected effective mass when allowing for filtering and the effective mass using unfiltered force and accelerometer data

\begin{tabular}{|c|c|c|c|c|c|}
\hline \multirow[b]{2}{*}{ Trial } & \multicolumn{2}{|c|}{ Filtered (kg) } & \multicolumn{2}{|c|}{ Corrected (kg) } & \multirow{2}{*}{$\begin{array}{l}\text { Unfiltered (kg) } \\
\text { Acc }\end{array}$} \\
\hline & Acc & Video & Acc & Video & \\
\hline 1 & 2.0 & 3.0 & 2.7 & 3.7 & 2.9 \\
\hline 2 & 1.6 & 1.8 & 2.3 & 2.5 & 2.0 \\
\hline 3 & 2.0 & 2.3 & 2.7 & 3.0 & 2.7 \\
\hline 4 & 1.6 & 2.1 & 2.3 & 2.8 & 3.4 \\
\hline 5 & 3.4 & 2.4 & 4.1 & 3.1 & 2.8 \\
\hline Mean & 2.1 & 2.3 & 2.8 & 3.0 & 2.8 \\
\hline
\end{tabular}

The effective mass from video data calculated with equal compression between layers (equation 5) was $2.0 \mathrm{~kg}$. For unequal compression (equation 7) the effective mass was $2.8 \mathrm{~kg}$. Half the mat mass was $3.05 \mathrm{~kg}$. Using the peak acceleration from the video and these effective masses the forces beneath the landing mat for the five trials were calculated and were compared to the force plate peak forces (Table 5). For the $2.0 \mathrm{~kg}$ effective mass peak vertical ground reaction force was underestimated within a $-0.5 \%$ to $-4.0 \%$ range. For the 
$2.8 \mathrm{~kg}$ forces were within a $-1.1 \%$ to $+3.7 \%$ range. For the $3.05 \mathrm{~kg}$ effective mass peak vertical ground reaction forces were overestimated within a $+0.1 \%$ to $+4.8 \%$ range.

Table 5. Percentage difference between calculated peak forces and actual peak forces

\begin{tabular}{|c|c|c|c|}
\multicolumn{1}{c}{ Trial } & $\begin{array}{c}\text { 2.0-kg effective } \\
\text { mat mass }\end{array}$ & $\begin{array}{c}2.8-\mathrm{kg} \text { effective } \\
\text { mat mass }\end{array}$ & $\begin{array}{c}3.05-\mathrm{kg} \text { effective } \\
\text { mat mass }\end{array}$ \\
\hline 1 & $-4.0 \%$ & $-1.1 \%$ & $+0.1 \%$ \\
\hline 2 & $-0.5 \%$ & $+3.7 \%$ & $+4.8 \%$ \\
\hline 3 & $-1.3 \%$ & $+1.6 \%$ & $+2.8 \%$ \\
\hline 4 & $-0.7 \%$ & $+2.3 \%$ & $+3.5 \%$ \\
\hline 5 & $-1.6 \%$ & $+1.3 \%$ & $+2.6 \%$ \\
\hline
\end{tabular}

Time is required for the shock wave produced at the top of the mat at impact to propagate through the mat to the force plate. The fact that the time delay was due to the sample landing mat was confirmed by striking the wooden box surrounding the mat with the impactor as this showed that there was no time delay between the strike and the force detection via the force plate. As the mat compresses during loading the shock transmission time decreases so that at maximum mat deformation the shock transmission time was reduced to approximately $3 \mathrm{~ms}$.

This transmission time is important when attempting to compare force and accelerometer data as the loading at the surface of the mat at time $t$ has not propagated to the bottom of the mat until time $t+\Delta t$. During the initial impact large forces can be produced at the surface of the mat and within the mat but not be registered at the force plate. This means that the calculation of the effective mass of the mat is not possible until the force plate has a reading and that for an accurate calculation of effective mass the time delay for the shock propagation needs to be known and accounted for. If the force plate values are used in conjunction with the kinematics of a gymnast this delay needs to be accounted for.

Using the unfiltered peak force and acceleration values does not take the shock propagation delays into account. However it was not possible to measure the delay time accurately other than at initial contact and peak force / acceleration. Uncertainty in the delay time introduces errors into the calculations of effective mass from force and acceleration data. Effective mass of the mat is required to calculate the difference in forces at the top and bottom of the mat. The effective mass of the mat being accelerated corresponds to a force that ranges from $480 \mathrm{~N}$ to $930 \mathrm{~N}$ and this cannot be ignored as it accounts for up to $12 \%$ of the peak force in the trials.

The high-speed video peak acceleration was on average within $2.5 \%$ of the accelerometer peak acceleration. Shock transmission times are a little harder to determine from high-speed video due to the difficulty in determining initial mat contact in a single frame but they were within $4 \mathrm{~ms}$ of the times determined from the accelerometer and force plate. High-speed video can also be used to describe the mat deformation. In these experiments the complex mat deformation may be characterised as a 3D rectangular depression near the mat centre with a triangular prism on either side, Method 1. Method 2 resulted in greater estimates of deformation volume than Method 1. It is likely that with a larger mat a more symmetrical deformation pattern will be present and therefore Method 2 may be more appropriate for determining deformation volume for a full size landing mat. In this case the effective mass calculations should use the area and relative accelerations for the deformation described by Method 2. Given that the complex mat deformations were finally characterized by these simpler geometries it is likely that using a single base area rather than two foot sized bases was sufficient for this study. 
Determining the effective mass from video data relied on assumptions and approximations. Despite this, using equation 5, along with the deformation as described in method 1, gave an effective mass of $2.8 \mathrm{~kg}$. This compares well with values for the effective mass calculated from force and acceleration measurements from Table 4 and indicates that the video method could determine a reasonable effective mass. These calculated mass values have only been reported to one decimal place and it is unlikely that the different effective mass methods truly give an identical result. Nonetheless, using the effective mass and the acceleration obtained by double differentiating positional data, the peak force measured at the bottom of the plate could be predicted to within 3.7\%. With displacement, velocity, acceleration, extent of deformation and material composition of the mat known the dynamic response of the mat can be calculated from video data alone. Obtaining the same data for the mat response to oblique impacts could allow the magnitude and direction of forces on a gymnast during landing to be calculated during competition. It could also allow the comparison of different mats when not all mats are available for testing in a laboratory.

\section{REFERENCES}

1. Abdel-Aziz, Y. I. and Karara, H. M. Direct linear transformation from comparator coordinates into object-spece coordinates. In Proceedings of the Symposium on CloseRange Photogrammetry. Falls Church, VA, pp.1-18, 1971.

2. Butler, R., Crowell, H., Davis, I. Lower extremity stiffness: implications for performance and injury. Clinical Biomech. 18: 511-517, 2003.

3. De Koning, J.J., Nigg, B.M., Gerritsen, K.G.M. Assessment of mechanical properties of area-elastic sport surfaces with video analysis. Med. Sci. Sports and Exerc. 29: 6641668, 1997.

4. Federation Internationale de Gymnastique - F.I.G. Apparatus Norms. F.I.G., Switzerland, F.I.G., 1996, pp.MAG 11.

5. Francis, P. R., Leigh, M., Berzins, A. Shock absorbing characteristics of floors used for dance exercise. Inter. J. Sports Bio. 4:282-305, 1998.

6. Frederick, E. Sport Shoes and Playing Surfaces: Their Biomechanical Properties, Human Kinetics Publishers,Champaign, IL. 1984, pp. vii-ix.

7. Fritz, M., Peikenkamp, K. Simulation of the influence of sports surfaces on vertical ground reaction forces during landing. Med. Bio. Engineering and Computing. 41:11-17, 2003.

8. Gatto, F., Swannell, P. and Neal, R. A force-indentation relationship for gymnastic mats. $J$. Bio. Engineering. 114:338-345, 1992.

9. Graff, K.F. Wave Motion in Elastic Solids. Dover Publications inc. 1991, pp 7-19. 1-8 edition 11975

10. McNitt-Gray, J. L., Hester, D., Mathiyakom, W. and Munkasy, B. Mechanical demand and multijoint control during landing depend on orientation of the body segments relative to the reaction force. J. Biomech. 34: 1471-1482, 2001.

11. McNitt-Gray, J.L., Yokoi, T., Millward, C. Landing strategy adjustments made by female gymnasts in response to drop height and mat composition. J. App. Biomech. 9:173190, 1993.

12. Takei, Y. Techniques used in performing handspring and salto forward tucked in gymnastics vaulting. Int. J. Sports Biomech. 4:260-281, 1988. 
13. Takei, Y. Three-dimensional analysis of handspring with full turn vault: deterministic model, coach’s beliefs and judges’ scores. J. App. Biomech. 14:190-211, 1998.

14. Yeadon, M.R., Nigg, B.M. A method for the assessment of area-elastic surfaces. Med. Sci. Sports and Exerc. 20:403-407, 1988. 